\title{
Complications of Intertrochanteric Rotational Osteotomy
}

\author{
Ethan M. Braunstein, M.D. ${ }^{1}$, Barbara N. Weissman, M.D. ${ }^{1}$, J. Leland Sosman, M.D. ${ }^{1}$, and \\ Michael Drew, M.D. ${ }^{2}$ \\ Departments of ${ }^{1}$ Radiology and ${ }^{2}$ Orthopaedics, Harvard Medical School, Brigham and Women's Hospital, Boston, Massachusetts, \\ USA
}

\begin{abstract}
Intertrochanteric anterior rotational osteotomy is a recently developed surgical procedure to treat osteonecrosis of the femoral head. We reviewed the radiographic findings in four cases to acquaint radiologists with the usual appearance of the procedure and to assess surgical complications. In all cases, immediate postoperative radiographs showed rotation of the necrotic portion of the femoral head anteriorly so that it was no longer weight-bearing. Clinical and radiologic follow-up ranged from 12 to 30 months. In this time, three patients developed complications, including nonunion of the osteotomy, further osteonecrosis with collapse of the femoral head, and worsening pain in the absence of progressive radiologic change.

Radiology provides an important means of assessing rotational osteotomy, particularly in demonstrating sufficient rotation of the femoral head to assure nonweight-bearing by diseased bone. Also, surgical complications such as nonunion and hardware loosening may be identified. Nevertheless, the patient may deteriorate clinically even in the absence of radiologic demonstration of disease progression, and the absence of radiographic change does not assure a successful surgical outcome.
\end{abstract}

Key words: Osteonecrosis - Femoral head - Osteotomy

Management of femoral head osteonecrosis ranges from core decompression to total hip arthroplasty. Recently Sugioka [6] described an intertrochanteric anterior rotational osteotomy, claiming that

Address reprint requests to: Dr. E.M. Braunstein, Department of Radiology, University of Michigan Hospitals, Ann Arbor, MI 48109, USA the procedure arrests the progression of disease and preserves enough bone stock to allow later femoral head replacement if this becomes necessary. Of 41 hips in Sugioka's series, only six pro-
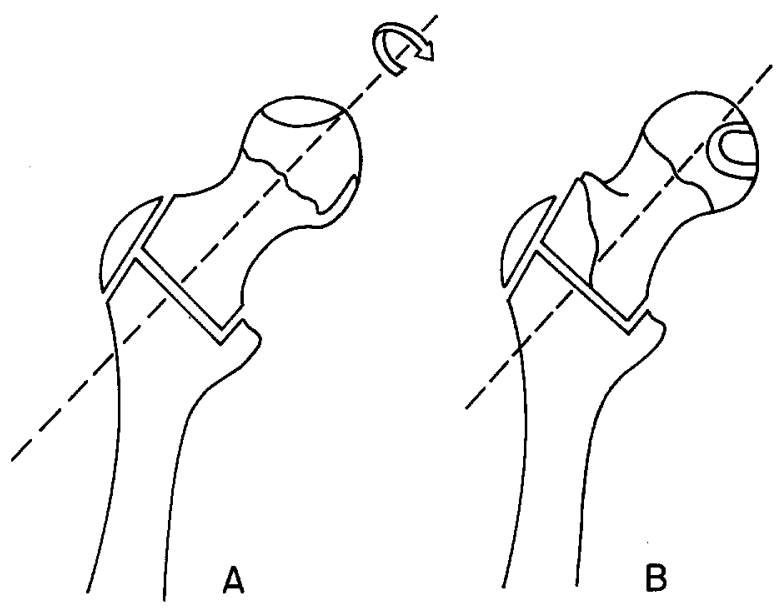

Fig. 1 A, B. Osteotomy and anterior rotation of femoral head to reposition osteonecrotic fragment away from weight-bearing portion of hip (after Sugioka [6]). A Preoperative position. B Postoperative position

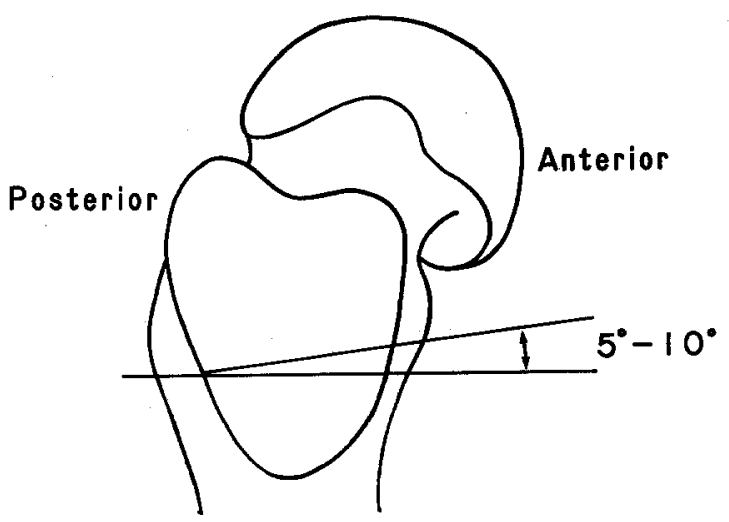

Fig. 2. Lateral view of hip, showing anterior wedge resected at osteotomy, causing varus repositioning of hip when femoral head is rotated (after Sugioka [6]) 

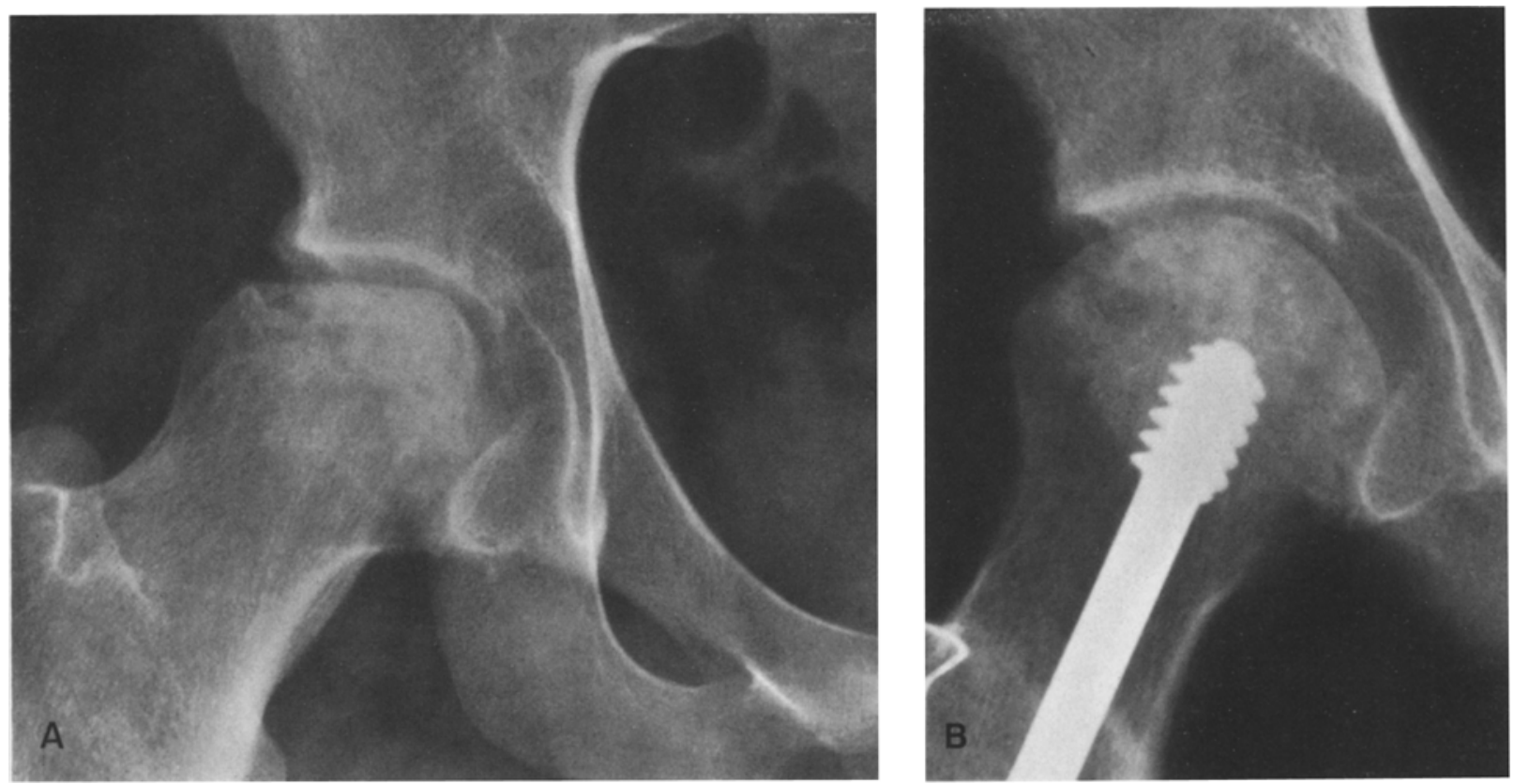

Fig. 3A, B. A 20-year-old man with Grade IV osteonecrosis secondary to steroid therapy for idiopathic thrombocytopenic purpura. A Preoperative anteroposterior radiograph. B Immediate postoperative anteroposterior radiograph showing that osteonecrotic segment has been rotated, and that weight-bearing area has a normal convexity. Notice placement of screw in the middle of the femoral head

Table 1. Radiologic grading of osteonecrosis

Grade I: $\begin{aligned} & \text { Early changes just visible; no flattening of femoral } \\ & \text { head }\end{aligned}$
Grade II: Flattening or beginning collapse of femoral head
Grade III: Severe collapse of head; normal width of cartilage
Grade IV: Advanced changes of head; cartilage narrowing

gressed to further collapse, regardless of the initial grade of disease. The procedure consists of rotating the femoral head anteriorly 45 to 90 degrees (Fig. 1). A wedge of bone may be removed (Fig. 2), so that the hip is in a slight varus position after the femoral head is rotated. Since osteonecrosis usually occurs in the anterior superior portion of the femoral head, the procedure rotates the area of involved bone out of the weight-bearing area of the hip. Thus, the posterior convexity of the femoral head, with normal articular cartilage, becomes the weight-bearing area, and further necrosis and collapse are theoretically prevented. We wish to acquaint radiologists with postoperative radiographic features of the "Sugioka osteotomy," in normal cases and in those with complications.

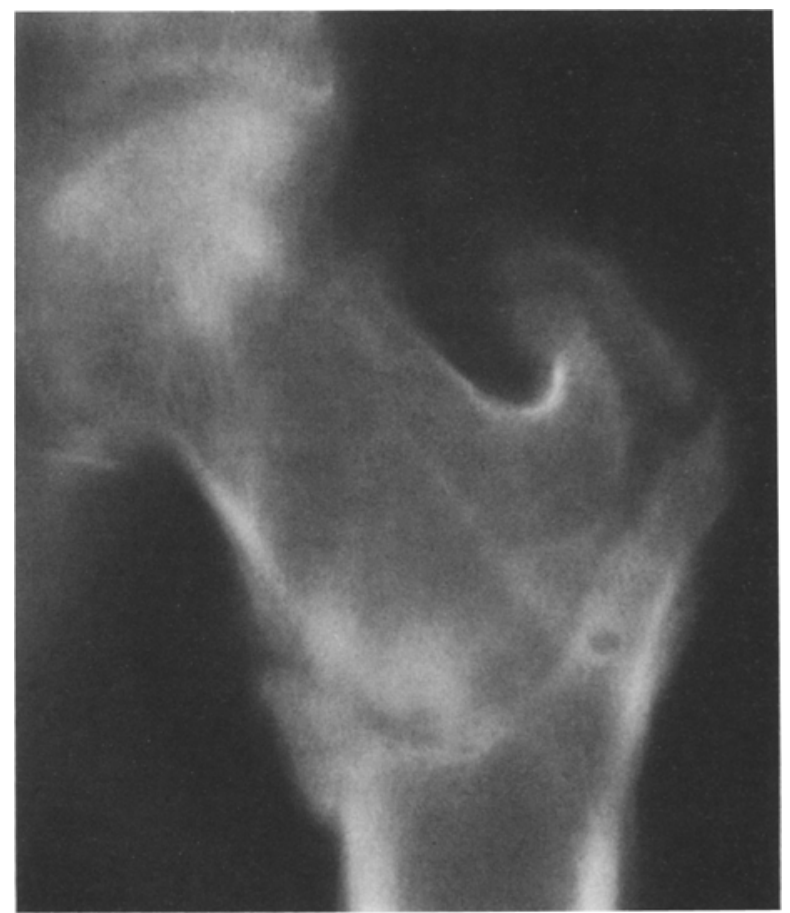

Fig. 4. A 48-year-old male with osteonecrosis presumed to be secondary to alcohol intake. Tomograms taken nine months postoperatively demonstrate nonunion of intertrochanteric osteotomy. The plate and screw had been removed at six months 

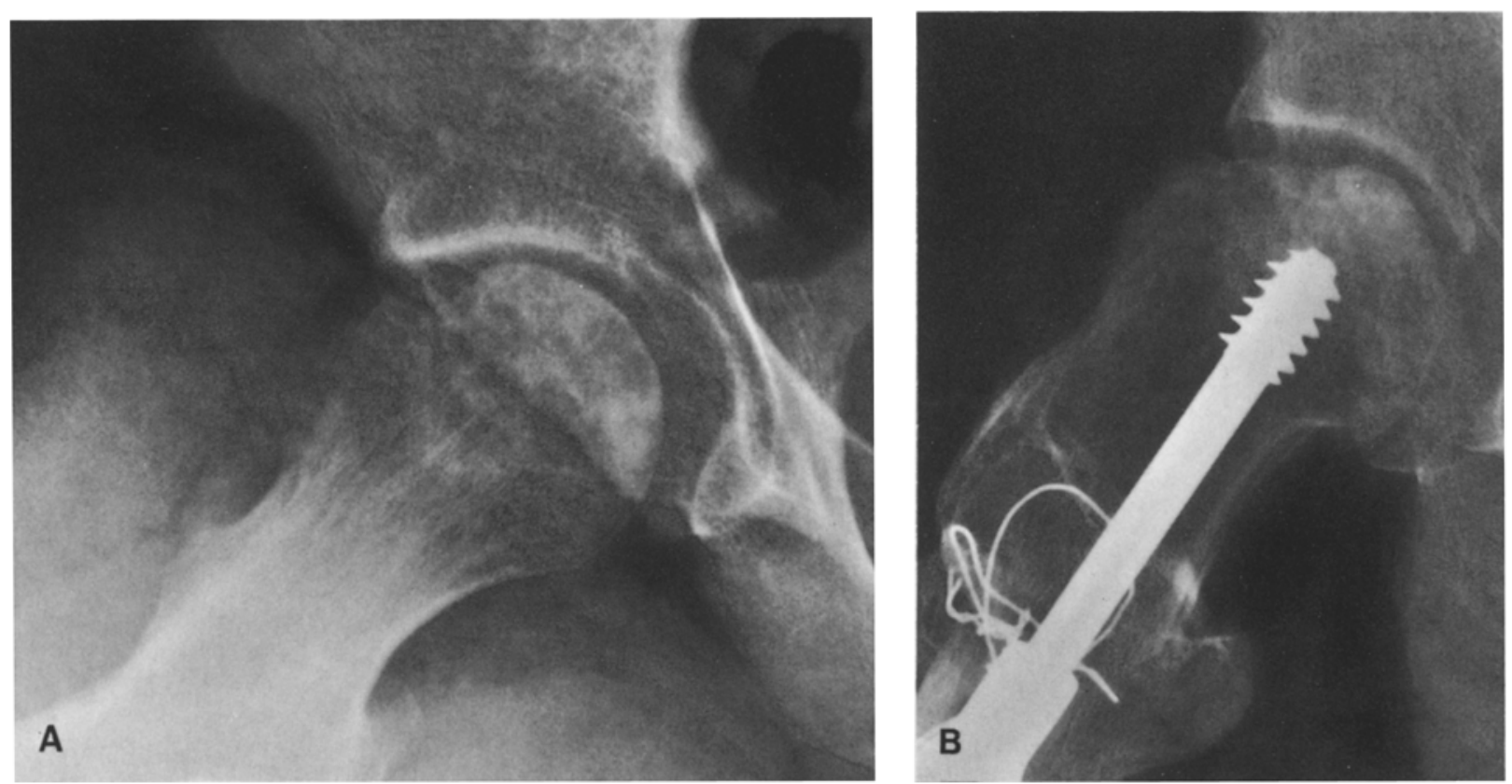

Fig. 5A, B. A 36-year-old male with osteonecrosis, presumed to be alcohol-related. A Frogleg lateral radiograph obtained one week before intertrochanteric rotational osteotomy. B Frogleg lateral radiograph 18 months after surgery. Notice progressive collapse of femoral head

\section{Clinical Material}

To demonstrate the range of complications of rotational osteotomy, we reviewed the radiographic findings in four patients. All were male, ranging in age from 20 to 49 years. In one patient, the disease was secondary to high-dose corticosteroid therapy for idiopathic thrombocytopenic purpura, the other cases were idiopathic, although possibly related to alcohol intake in two of the patients. Length of clinical and radiographic follow-up varied from 12 to 30 months. Clinically, one patient continues to have some pain, but the osteotomy healed and progression of femoral head collapse has been arrested. Another has had progressive collapse of the femoral head, but he has continued to take steroids. The third had a nonunion of the osteotomy, and the fourth underwent revision to a bipolar endoprosthesis due to continued pain one year after his original surgery.

\section{Radiologic Findings}

On preoperative radiographs, osteonecrosis was apparent in all patients. Two patients had Grade II changes, one patient had Grade III changes, and one patient had Grade IV changes (Fig, 3) according to a radiologic classification system used by Sugioka [6] (Table 1).

On radiographs obtained shortly after surgery, the rotation of the femoral head due to osteotomy should be apparent when projection is comparable to that of preoperative radiographs (Fig. 3). The necrotic segment of the femoral head should have been rotated anteriorly so that it is no longer weight-bearing. Regardless of later complications, this was true in all our patients.

By six months after surgery, there should be union at the osteotomy site. This was true in three of our cases, but the fourth had undergone painful nonunion. By this time, the typical findings of sclerosis, widening of the osteotomy gap and loosening of the hardware are seen (Fig. 4). No other complications were apparent radiographically in the other patients at this time. The osteotomies had healed, and there was no further fragmentation or collapse of the femoral heads.

On radiographs obtained one year after surgery, progressive osteonecrosis did develop in one case, with further femoral head collapse (Fig. 5). In another case, although there were no further radiographically demonstrable changes, the patient continued to experience hip pain. At two years, this patient underwent revision to a bipolar endoprosthesis in spite of the lack of further radiographic findings. The final patient has not sustained any further osteonecrosis or surgical complications after two years.

\section{Discussion}

These four cases represent the range of results seen at this institution in anterior intertrochanteric rotational osteotomy. The usefulness of this procedure 
remains controversial, as the clinical results in our patients attest. Indeed, femoral osteotomy of any kind for osteonecrosis or Legg-Perthes disease has achieved mixed results. Although good results have been reported with varus osteotomy, which contains the femoral head and decreases weight-bearing on the necrotic area [1-3], other authors have found no long-term differences between osteotomy and splinting [5]. Lloyd-Roberts et al. [4] suggest that the amounts of osteonecrosis and cartilage loss determine the outcome, and that varus-rotational osteotomy is useful only if severe deformity has not yet occurred.

Sugioka [6] maintains that simple varus or valgus osteotomy will not suffice for extensive lateral areas of osteonecrosis. The intertrochanteric rotational approach removes such areas from weightbearing and improves joint congruity by realignment; however it works less well for extensive bilateral disease. None of Sugioka's 13 patients with Grade I osteonecrosis had further collapse of the femoral head after surgery, although such deterioration did occur in 6 of 28 patients with Grades II-IV disease. Follow-up ranged from 18 months to four years. As with other osteotomies, the surgical outcome in these series proved to be better when the disease was less extensive.

Rotational osteotomy, compared with varus or valgus osteotomy, is technically very difficult. This may partially account for the complications described in this report. Technical errors include failure to rotate the femoral head sufficiently, failure to secure the osteotomy (causing non-union), and failure to assure retinacular blood supply to the femoral head, causing further osteonecrosis.

Radiologic examination provides an accurate means of assessing intertrochanteric osteotomy. Anteroposterior and lateral views show whether the hip has been rotated enough to allow weightbearing by uninvolved bone and cartilage. Further collapse of the femoral head, documented radiographically, is a predictor of clinical failure. Also nonunion, hardware loosening, and other technical complications of surgery may be assessed. Even without radiographic progression the patient may worsen clinically. The absence of radiographic changes assures a successful outcome for rotational osteotomy.

\section{References}

1. Canario AT, Williams L, Weintroub S, Catterall A, LloydRoberts GC (1980) A controlled study of the results of femoral osteotomy in severe Perthes disease. J Bone Joint Surg [Br] 62:438

2. Cordeiro EN (1980) Femoral osteotomy in Legg-CalvePerthes disease. Clin Orthop 150:69

3. Heikkinen E, Puranen J (1980) Evaluation of femoral osteotomy in the treatment of Legg-Calve-Perthes disease. Clin Orthop 150:60

4. Lloyd-Roberts GC, Catterall A, Salamon PB (1976) A controlled study of the indications for and the results of osteotomy in Perthes disease. J Bone Joint Surg [Br] 58:31

5. Marklund T, Tillberg B (1976) Coxa plana: A radiological comparison of the rate of healing with conservative measures and after osteotomy. J Bone Joint Surg [Br] 58:25

6. Sugioka Y (1978) Transtrochanteric anterior rotational osteotomy of the femoral head in the treatment of osteonecrosis affecting the hip. Clin Orthop 130:191 